\title{
Comparison of Media for Recovery of Verticillium dahliae from Soil
}

\author{
Z. Kabir, R. G. Bhat, and K. V. Subbarao, Department of Plant Pathology, University of California-Davis, c/o \\ United States Agricultural Research Station, Salinas 93905
}

\begin{abstract}
Kabir, Z., Bhat, R. G., and Subbarao, K. V. 2004. Comparison of media for recovery of Verticillium dahliae from soil. Plant Dis. 88:49-55.

Polygalacturonic acid (PGA) is an important constituent of Sorensen's NP-10 medium (NP-10) for estimating the population density of Verticillium dahliae in soil. Different types of PGA are available, but not all of them favor the growth of $V$. dahliae. Unavailability of PGA sodium salt from orange (P-1879) has created an unprecedented problem for the quantification of microsclerotia (MS) of $V$. dahliae in soil. The PGA from orange (P-3889) that is now available does not support the growth of $V$. dahliae. Therefore, experiments were conducted to optimize the use of NP-10 prepared with P-3889 and various concentrations of NaOH. NP-10 with P-3889 amended with eight concentrations of $\mathrm{NaOH}$ were compared with NP-10 prepared from PGA sodium salt from orange (P-1879, now discontinued) and citrus (P-3850) along with cellophane and Napectate media for recovery of MS from soil and growth of $V$. dahliae on the media. Seven soils were assayed for MS, and eight isolates of $V$. dahliae were evaluated for growth and production of MS. Concentrations of $\mathrm{NaOH}>0.035 \mathrm{~N}$ and $<0.02 \mathrm{~N}$ in NP-10 with P-3889 reduced mycelial growth, microsclerotial production, and recovery of MS from soils. Similarly, NP-10 with P3850 alone, cellophane, and Na-pectate media had significantly reduced growth on media and recovery of $V$. dahliae from soils. The NP-10 with P-3889 and $0.025 \mathrm{~N} \mathrm{NaOH}$ consistently yielded numbers of $V$. dahliae MS from soil samples and supported the growth and production of MS similar to the NP-10 with P-1879. The medium developed in this study can serve as a direct replacement for the original NP-10 that was developed nearly three decades ago, an important component of which is no longer available.
\end{abstract}

Verticillium dahliae Kleb. is a ubiquitous soilborne fungus causing wilt diseases on many economically important crops $(2,13,14,17)$. The pathogen forms multicelled, melanized, resting bodies called microsclerotia (MS), which persist in the soil for many years $(14,20)$. MS in soil are the primary source of inoculum for $V$. dahliae infections in host plants (21). Incidence of Verticillium wilt in herbaceous hosts is generally proportional to the number of MS in soil $(11,12,19)$, but can vary considerably depending on the cultivar and crop $(5,6,8,21)$. For example, in cauliflower, 4 MS $\mathrm{g}^{-1}$ of dry soil can cause $16 \%$ wilt incidence but $10 \mathrm{MS} \mathrm{g}^{-1}$ of soil causes $50 \%$ wilt (21). In tomato, $0.5 \mathrm{MS} \mathrm{g}^{-1}$ of soil causes $50 \%$ wilt incidence but $6 \mathrm{MS} \mathrm{g}^{-1}$ of soil causes $100 \%$ wilt (5). In strawberry, however, as little as $0.3 \mathrm{MS} \mathrm{g}^{-1}$ of soil causes $5 \%$ wilt and just $2 \mathrm{MS} \mathrm{g}^{-1}$ of soil can cause $100 \%$ wilt (6). Therefore, accurate quantification of MS in the soil is essential to make planting decisions, to predict the risk of disease in a given field, and to manage Verticillium wilt in commercial fields.

Corresponding author: K. V. Subbarao

E-mail: kvsubbarao@ucdavis.edu

Accepted for publication 5 September 2003.

Publication no. D-2003-1111-01R

(C) 2004 The American Phytopathological Society
Three techniques have been employed widely to quantify MS from soil: wet sieving, soil dilution plating, and soil impaction plating using an Anderson sampler (10). Among these techniques, the Anderson sampler technique is considered to be the least biased method $(3,18)$. With this technique, a known mass of soil is uniformly spread on a petri-plate with Sorensen's NP-10 semiselective medium (15) using the Anderson sampler. After a 3week incubation in the dark, microsclerotial colonies are enumerated under a stereoscope.

Polygalacturonic acid (PGA) was identified as an important constituent in the semiselective medium for the growth of $V$. dahliae MS nearly three decades ago. Until recently, sodium salt of PGA from orange (P-1879) (Sigma-Aldrich, St. Louis) had been optimized for use in Sorensen's modified NP-10 medium (15) and has been the most widely used medium for soil assays of $V$. dahliae. About 3 years ago, SigmaAldrich discontinued this product and this has rendered accurate quantification of MS in soil using NP-10 medium difficult. One type of PGA that is currently available from Sigma-Aldrich is a PGA-sodium salt from citrus (P-3850). In preliminary studies, the use of this product in Sorensen's NP-10 medium greatly underestimated the number of MS present in soil and drastically reduced the production of MS. Another type of PGA that is available from Sigma-Aldrich is derived from orange (P-
3889); however, in preliminary studies, NP-10 medium prepared with this PGA did not support the growth of $V$. dahliae. The $\mathrm{pH}$ of NP-10 with P-3889 was significantly lower than NP-10 with P-1879; therefore, we decided to amend the medium with various concentrations of $\mathrm{NaOH}$ to optimize the NP-10 for the growth and recovery of $V$. dahliae.

A number of substrate-specific methods have been developed and tested to estimate the MS recovery from soils. Among these methods, cellophane (1) and Na-pectate (3) media are considered effective, but never have been compared with the NP-10 medium for growth of $V$. dahliae and recovery of MS. The objectives of this study were to optimize NP-10 prepared with P-3889 using various concentrations of $\mathrm{NaOH}$ and compare this medium with currently available media for growth and production of MS by isolates of $V$. dahliae from various hosts, and to compare the media for soil assays of $V$. dahliae MS. A preliminary report has been published (9).

\section{MATERIALS AND METHODS}

Media preparation. Sorensen's NP-10 semiselective medium (15) was prepared with PGA-sodium salt from orange (P1879; molecular weight 4,000 to 6,000 ), PGA-sodium salt from citrus (P-3850; molecular weight of pectin purified to make P-3850 is 50,000 to 150,000 ), or PGA from orange (P-3889; molecular weight 4,000 to 6,000 ) amended with $\mathrm{NaOH}$. The NP-10 medium prepared with P-3889 was amended with concentrations of $0.01,0.015,0.02,0.025,0.03,0.035$, 0.045 , and $0.055 \mathrm{~N} \mathrm{NaOH}$ (Table 1). Each NP-10 medium consisted of two parts autoclaved separately. In the first part, $5 \mathrm{~g}$ of PGA (if PGA used was P-3889, then various amounts of $\mathrm{NaOH}$ were added to give the concentrations of $\mathrm{NaOH}$ listed above) in $500 \mathrm{ml}$ of distilled water was autoclaved at $121^{\circ} \mathrm{C}$ for $15 \mathrm{~min}$ and cooled to $50^{\circ} \mathrm{C}$. In the second part, $15 \mathrm{~g}$ of BactoAgar (0140-01; Difco Laboratories, Detroit), $1 \mathrm{~g}$ of $\mathrm{KNO}_{3}$ (P-8394; Sigma-Aldrich), $1 \mathrm{~g}$ of $\mathrm{KH}_{2} \mathrm{PO}_{4}$ (P-5379; SigmaAldrich), $0.5 \mathrm{~g}$ of $\mathrm{KCl}$ (P-4504; SigmaAldrich), $0.5 \mathrm{~g}$ of $\mathrm{MgSO}_{4} \cdot 7 \mathrm{H}_{2} \mathrm{O}(\mathrm{M}-1880$; Sigma-Aldrich), $0.5 \mathrm{ml}$ of Tergitol (NP-10; Sigma-Aldrich), and $500 \mathrm{ml}$ of distilled water (with a magnetic stir bar) were autoclaved at $121^{\circ} \mathrm{C}$ for $15 \mathrm{~min}$ and cooled to $50^{\circ} \mathrm{C}$. After the second part equilibrated to $50^{\circ} \mathrm{C}$, chloramphenicol (C-0378; SigmaAldrich), streptomycin sulfate (S-6501; Sigma-Aldrich), and chlorotetracycline 
$\mathrm{HCl}$ (C-4881; Sigma-Aldrich) at $50 \mathrm{mg}$ liter $^{-1}$ each were added. When the antibiotics were completely dissolved, the two parts were mixed and placed on a magnetic stirrer. Each medium was dispensed $(15 \mathrm{ml}$ per dish) into 90-mm petri dishes immediately.

In addition to the above media, cellophane (1) and Na-pectate (3) media were used for soil assays of $V$. dahliae MS (Table 1). The $\mathrm{pH}$ of all media was measured using a $\mathrm{pH}$ meter (Fisher Scientific, Santa Clara, CA) when the media temperature ranged between 39 and $45^{\circ} \mathrm{C}$ after each batch was prepared. Each repetition was considered as a replication in the analysis of variance to determine $\mathrm{pH}$ differences between the media using the general linear model (GLM) procedure in SAS (SAS Institute, Cary, NC). Fisher's protected least significant difference (LSD) test was used to compare mean $\mathrm{pH}$ between the different media.

Mycelial growth and production of MS by isolates of $\boldsymbol{V}$. dahliae. Isolates of $V$. dahliae from eight hosts (Table 2) were evaluated on all the media described above (Table 1). Single-spore isolations were made; cultures were purified and maintained on potato-dextrose agar (PDA) slants at $4^{\circ} \mathrm{C}$. Three plates of each medium were inoculated centrally with a $0.4-\mathrm{cm}$ diameter agar disk from the advancing margins of 2-week-old cultures of each isolate on PDA. All plates were incubated for 4 weeks at room temperature $(23 \pm$ $\left.1^{\circ} \mathrm{C}\right)$. Colony diameters on all plates and the diameter of the culture with MS were measured at $7,14,21$, and 28 days of incubation. The experiment was conducted three times. Repeated measures analysis of variance was used to test radial growth of $V$. dahliae colonies and the portion of the medium with MS in cultures (SAS release 8.0; SAS Institute). Means and corresponding standard errors were computed for each treatment and sampling time.

Length and width of MS on various media. The optimum range of $\mathrm{NaOH}$ con- centration in NP-10 medium containing P3889 that supported the growth of $V$. dahliae identical to the NP-10 medium containing P-1879 was determined in the above experiment. The morphology of $V$. dahliae MS from several isolates were compared on A, B, E, G, K, and M media (Table 1). Only three isolates were selected for length and width of MS measurement based on their growth and uniformity of MS formation in NP-10 with P-1879. To demonstrate that isolates from different crops have consistent growth on these media, cultures of $V$. dahliae isolates from chili pepper (VdCa.36), lettuce (VdLs.17), and oilseed rape (VdBno.188) were produced on each of the above media as described above. The experiment was conducted twice. Each time, 10 agar plugs (4-mm-diameter) from each of the medium-isolate combinations were placed on a slide and spread with a cover slip. Measurements of the length and width of MS were made on 100 MS from 28-day-old cultures on each medium under a stereoscope at $\times 100$. The length and width of MS data were analyzed using the SAS GLM procedure (SAS Institute). Data within each medium-isolate combination were pooled from the two experiments because there was no experiment-isolate interaction. Means and standard error of the mean were computed.

Production of MS. The variation in the number of MS produced on different semiselective media (A, B, E, G, K, and $\mathrm{M}$; Table 1) was determined in three iso- lates (chili pepper (VdCa.36), lettuce (VdLs.17), and oilseed rape (VdBno.188)) of $V$. dahliae (Table 2). These isolates were selected based on their growth in the above experiment. Agar plugs (4-mm-diameter) from 28-day-old cultures of each mediumisolate combination were placed on a slide and uniformly spread with a cover slip. The number of MS was counted in five microscopic fields with a stereoscope at $\times 100$ and, for each isolate, 20 agar plugs per medium were examined and each agar plug was considered as a replication. The experiment was repeated once. Analysis of variance was done using the GLM procedure of SAS to test the effects of semiselective media on MS production. Means and standard errors of the mean were computed.

Comparison of media for assay of $\boldsymbol{V}$. dahliae MS in field soil. Ten soil samples each were collected to a depth of $10 \mathrm{~cm}$ from seven different commercial lettuce and strawberry fields in the Salinas Valley with moderate to high incidence of Verticillium wilt on the respective crops (Table 1). The 10 soil samples from each field were bulked for assay of individual field soils and air-dried on greenhouse benches. The air-dried soil was passed through a 425- $\mu \mathrm{m}$ (40-mesh) sieve. Sieved soil (10 g) from each sample was placed in a screwcapped plastic vial and $2.5 \mathrm{ml}$ of methionine (M-9500; Sigma-Aldrich; $0.0075 \mathrm{~g} \mathrm{ml}^{-1}$ of distilled $\mathrm{H}_{2} \mathrm{O}$ ) solution was dispensed into the vial. The vials then were incubated at $30^{\circ} \mathrm{C}$ for a week and air-dried at room

Table 2. Verticillium dahliae isolates used in this study with their hosts and geographical location

\begin{tabular}{|c|c|c|}
\hline Isolate number & Original host & Source \\
\hline VdCs. 80 & Artichoke (Cynara scolymus L.) & California \\
\hline VdBoc.74 & Cabbage (Brassica oleracia var. capitata $\mathrm{L}$.) & California \\
\hline VdBob.70 & Cauliflower (Brassica oleracia var. botrytis L.) & California \\
\hline VdCa.36 & Chili pepper (Capsicum аппиит L.) & California \\
\hline VdLs.17 & Lettuce (Lactuca sativa L.) & California \\
\hline VdMp.89 & Mint (Mentha $\times$ piperita L. $)$ & Oregon \\
\hline VdBno.188 & Oilseed rape (Brassica napus L.) & Germany \\
\hline VdFca.1 & Strawberry (Fragaria $\times$ ananassa Duchesne) & California \\
\hline
\end{tabular}

Table 1. Effects of media on the quantification of microsclerotia from different soils

\begin{tabular}{|c|c|c|c|c|c|c|c|c|}
\hline \multirow[b]{2}{*}{ Media $^{\mathbf{a}}$} & \multirow[b]{2}{*}{ Media pH } & \multicolumn{7}{|c|}{ Number of microsclerotia (MS) per gram of dry soil } \\
\hline & & Soil 1 & Soil 2 & Soil 3 & Soil 4 & Soil 5 & Soil 6 & Soil 7 \\
\hline A & 4.81 & 25 & 149 & 138 & 411 & 343 & 573 & 714 \\
\hline B & 7.49 & 4 & 14 & 112 & 166 & 108 & 158 & 141 \\
\hline C & 2.63 & 1 & 0 & 3 & 1 & 2 & 0 & 0 \\
\hline D & 2.91 & 1 & 2 & 4 & 2 & 9 & 11 & 12 \\
\hline E & 3.16 & 2 & 45 & 41 & 38 & 89 & 92 & 110 \\
\hline $\mathrm{F}$ & 3.86 & 3 & 34 & 64 & 162 & 215 & 348 & 287 \\
\hline G & 5.03 & 26 & 154 & 158 & 374 & 425 & 576 & 654 \\
\hline $\mathrm{H}$ & 5.68 & 27 & 125 & 139 & 366 & 369 & 628 & 635 \\
\hline I & 6.99 & 16 & 130 & 103 & 319 & 350 & 494 & 555 \\
\hline $\mathrm{J}$ & 7.67 & 12 & 77 & 105 & 313 & 302 & 527 & 506 \\
\hline K & 8.41 & 11 & 53 & 96 & 272 & 267 & 457 & 470 \\
\hline $\mathbf{L}$ & 7.57 & 23 & 83 & 75 & 223 & 184 & 384 & 380 \\
\hline $\mathrm{M}$ & 4.87 & 1 & 48 & 64 & 132 & 159 & 225 & 177 \\
\hline LSD & 0.19 & 10 & 47 & 60 & 131 & 135 & 147 & 164 \\
\hline
\end{tabular}

${ }^{a}$ Media are: A, polygalacturonic acid (PGA) (P-1879); B, PGA (P-3850); C, PGA (P-3889); D, P-3889 + 0.01 N NaOH; E, P-3889 +0.015 N NaOH; F, P$3889+0.02 \mathrm{~N} \mathrm{NaOH} ; \mathrm{G}, \mathrm{P}-3889+0.025 \mathrm{~N} \mathrm{NaOH} ; \mathrm{H}, \mathrm{P}-3889+0.03 \mathrm{~N} \mathrm{NaOH} ; \mathrm{I}, \mathrm{P}-3889+0.035 \mathrm{~N} \mathrm{NaOH} ; \mathrm{J}, \mathrm{P}-3889+0.045$ N NaOH$; \mathrm{K}, \mathrm{P}-3889+$ $0.055 \mathrm{~N} \mathrm{NaOH}$; L, cellophane; and M, Na-pectate. PGA was added to the Sorensen's NP-10 medium (15); Na-pectate and cellophane were added to the media as describe in Butterfield and DeVay (3) and Ashworth et al. (1). LSD = least significant difference $(P \leq 0.05)$. 
temperature $\left(23 \pm 1^{\circ} \mathrm{C}\right)$ for a week. All airdried soil from the vial then was poured into a mortar and pulverized gently to break the clods. The pulverized soil was plated onto five plates each, using the Anderson sampler technique on 13 different media (Table 1). The plates were incubated for 3 weeks in the dark at room temperature $\left(23 \pm 1{ }^{\circ} \mathrm{C}\right)$. Following incubation, the surface of each plate was gently washed under a stream of water to remove soil. The numbers of microsclerotial colonies of $V$. dahliae on each plate were counted under a stereoscope at $\times 10$ to $\times 20$. The density of MS was expressed as the number of propagules per gram of dry soil. The experiment was conducted four times and served as replications. Analysis of variance was conducted for MS recovery from the soils in different media using GLM in SAS. Fisher's protected LSD test was used to compare mean MS recovery on different media.

Germination of the recovered MS from two soils also was tested on the cellophane and Na-pectate media, and NP-10 containing P-1879, P-3850, or P-3889 amended with $0.025 \mathrm{~N} \mathrm{NaOH}$. Single colonies of $V$. dahliae MS from soils 4 and 5 (Table 2) from corresponding media were transferred to 10 plates of each medium and incubated at room temperature. The MS were considered germinated if a new colony of $V$. dahliae developed on the plated media. The number of plates yielding $V$. dahliae colonies was expressed as a percentage of the 10 plates examined. The experiment was conducted three times. Data were log transformed prior to analysis to normalize variance. Analysis of variance was conducted and Fisher's protected LSD was used to compare germination of MS on the selective media.
Effects of the volume of soil plated on MS recovery. Even though the amount of soil for plating in the Anderson sampler technique was standardized several decades ago (3), because of the contemporary changes in the composition of NP-10 medium, the assay procedure had to be standardized anew. Therefore, the influence of plated soil volume on the efficiency of MS recovery in $V$. dahliae-infested soil was determined. Ten soil samples collected from two commercial lettuce fields in the central coast of California were pooled and air-dried in paper bags for 2 to 3 weeks in a greenhouse. The soils were processed as described above and each soil sample was plated onto five plates of medium $\mathrm{G}$ (Table 1) at the rate of one scoop $(33 \mathrm{mg})$, two scoops $(67 \mathrm{mg})$, three scoops (100 mg), four scoops $(133 \mathrm{mg}$ ), five scoops (166 $\mathrm{mg}$ ), and six scoops (200 mg) per plate.
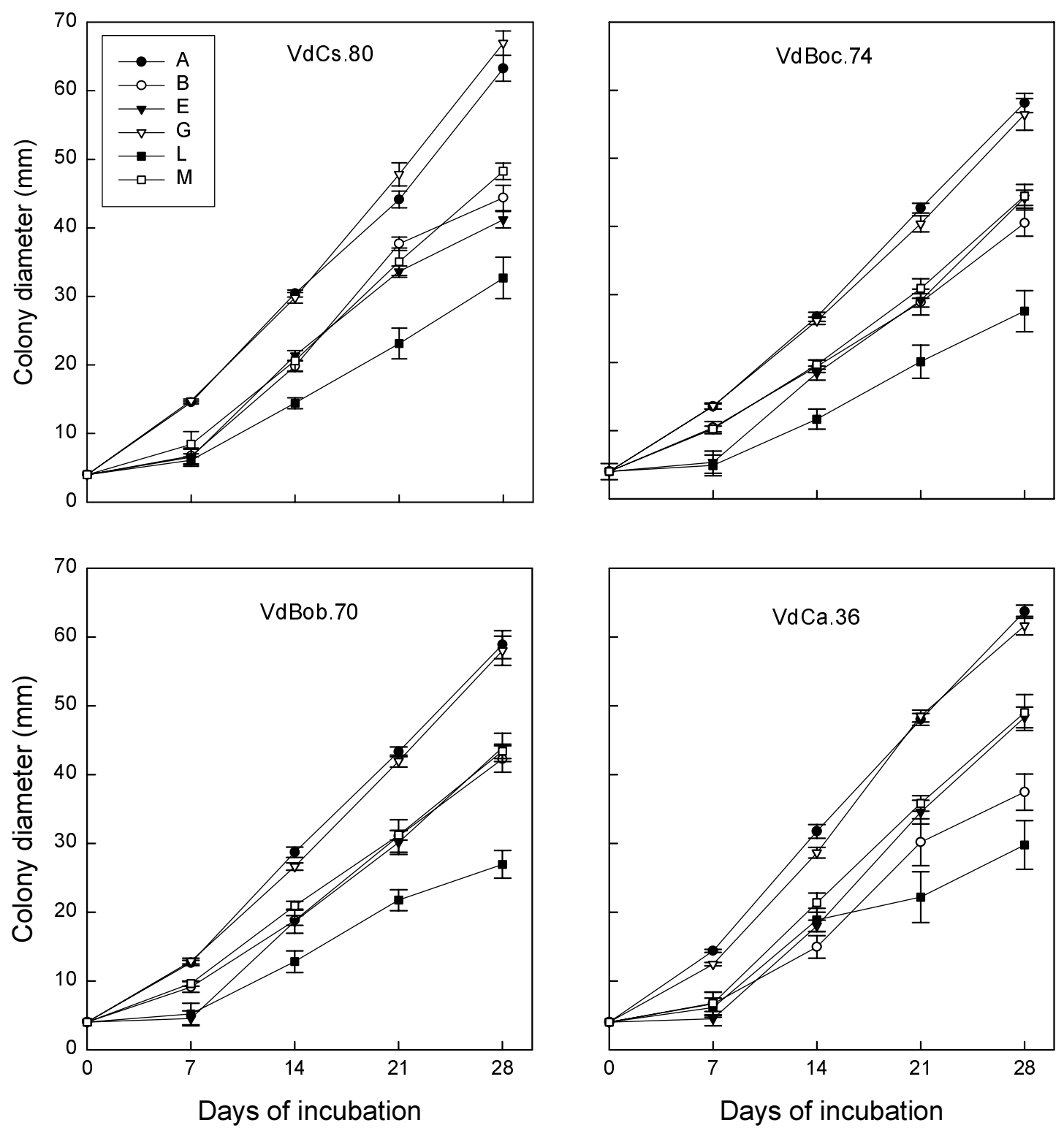

Fig. 1. Radial growth of Verticillium dahliae isolates from artichoke (VdCs.80), cabbage (VdBoc.74), cauliflower (VdBob.70), and chili pepper (VdCa.36) in different media after various days of incubation. The media are A, NP-10 with P-1879; B, NP-10 with P-3850; E, NP-10 with P-3889 and 0.015 N NaOH; G, NP-10 with P-3889 and 0.025 N NaOH; L, cellophane; and M, Na-pectate. Vertical bars represent the standard errors of the mean. 
The plates were incubated for 3 weeks in the dark at room temperature $\left(23 \pm 1{ }^{\circ} \mathrm{C}\right)$. Following incubation, the surface of each plate was washed gently under a stream of water to remove soil. The number of microsclerotial colonies of $V$. dahliae on each plate was counted under a stereoscope at $\times 10$ or $\times 20$. The density of MS was expressed as the number of propagules per gram of dry soil. The experiment was conducted four times; each experiment served as a replication. Analysis of variance was performed on the data to determine if soil volume affected recovery of MS. Means and standard errors were computed by pooling the data from the four experiments, because the experiment-treatment interaction was not significant.

\section{RESULTS}

pH of the media. The pH of NP-10 medium containing P-3889 was the lowest, cellophane or NP-10 containing P-3850 was the highest, and Na-pectate medium and NP-10 containing P-1879 were intermediate (Table 1). The NP-10 medium containing P-3889 amended with increasing concentrations of $\mathrm{NaOH}$ incrementally increased the $\mathrm{pH}$ and reached 8.41 with the highest concentration of $\mathrm{NaOH}$ included in the study ( $\mathrm{D}$ to $\mathrm{K}$ in Table 1 ). The $\mathrm{pH}$ in NP-10 containing P-3889 amended with $0.025 \mathrm{~N} \mathrm{NaOH}$ was 5.03, which was very close to the $\mathrm{pH}$ in NP-10 medium containing $\mathrm{P}-1879$ (original PGA; $\mathrm{A}$ and $\mathrm{G}$ in Table 1).

Effect of media on mycelial growth. The NP-10 medium containing P-3889 alone did not support the growth of any $V$. dahliae isolates. However, mycelial growth was observed in all NP-10 media containing P-3889 and amended with different concentrations of $\mathrm{NaOH}$. The mycelial growth progressively increased as the con- centration of $\mathrm{NaOH}$ in the medium increased from 0.01 to $0.025 \mathrm{~N}$, but then declined with further increase in the concentration of $\mathrm{NaOH}$ (data not shown). Colony diameters of all isolates of $\mathrm{V}$. dahliae in NP-10 with P-3889 and $0.025 \mathrm{~N}$ $\mathrm{NaOH}$ were identical to that on NP-10 added with P-1879 $(P>0.05)$ and were significantly greater than on all other tested media (Figs. 1 and 2). At the end of the 4week incubation, colony diameters of all $\mathrm{V}$. dahliae isolates in these two media were identical and were significantly greater than in all other media.

Colony diameters of all isolates except VdMp.89 were smallest in the cellophane medium (Figs. 1 and 2). Overall colony diameters of the oilseed rape (VdBno.188) isolate was smaller than all other isolates on all media tested.

Number of MS. The number of MS produced by three isolates of $V$. dahliae in
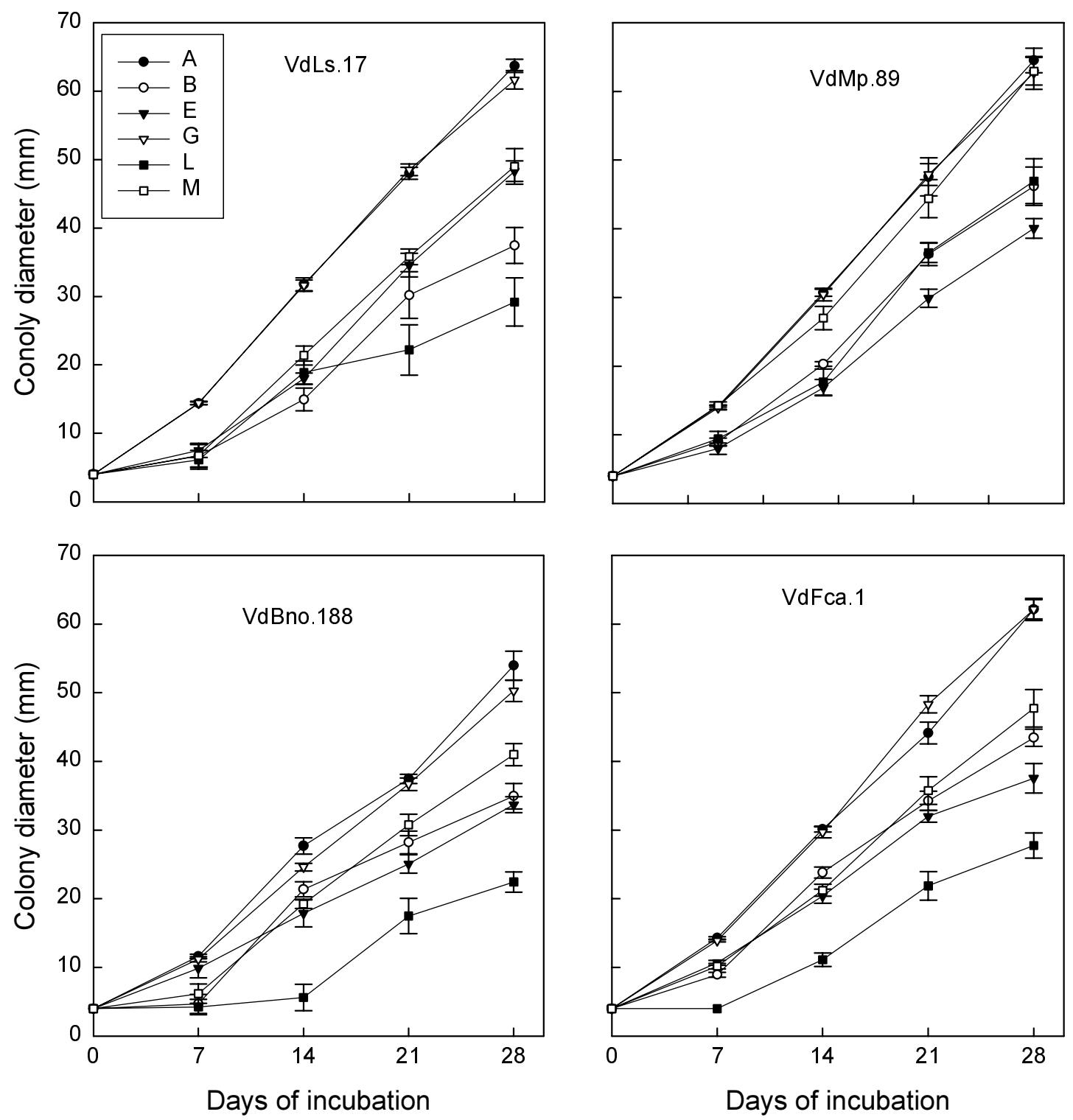

Fig. 2. Radial growth of Verticillium dahliae isolates from lettuce (VdLs.17), mint (VdMp.89), oilseed rape (VdBno.188), and strawberry (VdFca.1) in different media after various days of incubation. The media are A, NP-10 with P-1879; B, NP-10 with P-3850; E, NP-10 with P-3889 and 0.015 N NaOH; G, NP-10 with P-3889 and $0.025 \mathrm{~N} \mathrm{NaOH}$; L, cellophane; and M, Na-pectate. Vertical bars represent standard errors of the mean. 
4-mm-diameter agar plugs of different media is summarized in Figure 3. All isolates produced MS in all media tested except for VdLs.17, which failed to produce MS on NP-10 with P-3889 and either 0.015 or $0.055 \mathrm{~N} \mathrm{NaOH}$ (Fig. 3). The latter two media also supported the fewest number of MS by the other two isolates tested. In all media tested, isolate $\mathrm{VdCa} .36$ produced higher numbers of MS than isolates VdLs.17 and VdBno.188. The numbers of MS produced by all three isolates in the NP-10 with either P-1879 or P-3889 and $0.025 \mathrm{~N} \mathrm{NaOH}$ were nearly identical. The numbers of MS in these two media were significantly greater than in all other media except for VdLs.17 on Na-pectate medium.

Effect of media on microsclerotial length and width. The length and width of MS produced by isolate VdBno.188 were significantly greater than those produced by VdCa.36 and VdLs.17 on all media (Fig. 4). Both length and width of MS were not significantly different in NP-10 medium containing $\mathrm{P}-1879$ and NP-10 with P-3889 and $0.025 \mathrm{~N} \mathrm{NaOH}$, but were significantly greater than in all other media.

Recovery of MS from soils. No microsclerotial colonies were observed on NP-10 medium containing P-3889 alone in three tested soils. In the other four soils, less than $1 \%$ of microsclerotial colonies were recovered. The recovery of MS of $V$. dahliae from soils on NP-10 medium containing P-3889 improved with the addition of $\mathrm{NaOH}$ to the medium, and progressively increased as the concentration of $\mathrm{NaOH}$ increased from 0.01 to $0.03 \mathrm{~N}$, but then declined with further increase in the concentration of $\mathrm{NaOH}$ (Table 1). However, the numbers of MS recovered in the NP-10 medium containing P-3889 amended with 0.025 to $0.045 \mathrm{~N} \mathrm{NaOH}$ were not significantly different (Table 1) in five soils. The recovery of MS from soils on the NP-10 medium containing P-3889 and $0.025 \mathrm{~N}$ $\mathrm{NaOH}$ was identical to that of NP-10 containing P-1879 in all soils (Table 1). The NP-10 medium containing P-3850, Napectate, and cellophane media supported the recovery of $V$. dahliae MS but at significantly lower numbers than NP-10 containing either P-1879 or P-3889 with 0.025 to $0.045 \mathrm{~N} \mathrm{NaOH}$ (Table 1). Recovery of MS from soils by NP-10 containing P3850 or Na-pectate was 9 to $81 \%$ and 4 to $46 \%$, respectively, relative to NP-10 containing P-1879. However, the recovery by NP-10 containing P-3889 with $0.025 \mathrm{~N}$ $\mathrm{NaOH}$ was 91 to $123 \%$ of that in NP-10 containing P-1879 (Table 1).

Similarly, the germination of MS recovered from soils 4 and 5 also was highest on NP-10 containing P-1879 (63 and 60\% for soils 4 and 5, respectively) or P-3889 with $0.025 \mathrm{~N} \mathrm{NaOH}$ (57 and 63\% for soils 4 and 5 , respectively). Germination was least on the cellophane medium (17 and 13\% for soils 4 and 5 , respectively), and inter- mediate on Na-Pectate (48 and $30 \%$ for soils 4 and 5 , respectively) and NP-10 containing P-3850 (37 and 33\% for soils 4 and 5 , respectively).

Effect of amount of soil plated on recovery of MS. The number of MS per gram of soil was not proportional to the amount of the soil plated on NP-10 medium in both tested soils (Fig. 5). In soil A, significantly lower numbers of MS were recovered when six scoops (200 mg) of soil were plated on each plate than at all other soil volumes plated. However, in soil $\mathrm{B}$, the numbers of MS per gram of soil were significantly lower when one scoop $(33 \mathrm{mg}$ ) of soil was plated. The numbers of MS increased when up to three scoops $(100 \mathrm{mg})$ of soil was plated on each plate and declined thereafter in both tested soils (Fig. 5).

\section{DISCUSSION}

Soil assays of $V$. dahliae MS are an integral part of any laboratory working on the detection, epidemiology, and control of this pathogen on the myriad crops that it infects. The mainstay of soil assays in a majority of the laboratories has been the NP10 semiselective medium for the recovery of microsclerotial colonies of $V$. dahliae (15). The critical component in the NP-10 semiselective medium has been the sodium salt of PGA (P-1879), hitherto supplied by Sigma-Aldrich. Discontinuation of this product by the company has necessitated the identification of alternative sources of PGA for efficient recovery of $V$. dahliae MS from soil. As demonstrated in this study, the numbers of MS recovered from different soils, the size of MS of different isolates, and the growth rates of these isolates on NP-10 with P-3889 and $0.025 \mathrm{~N}$ $\mathrm{NaOH}$ were identical to those obtained on NP-10 with P-1879. Thus, NP-10 with P3889 and $0.025 \mathrm{~N} \mathrm{NaOH}$ is an ideal alternative to NP-10 with P-1879.

NP-10 with P-3889 alone did not allow the recovery of MS from the soil and did not support the growth of $V$. dahliae isolates tested and, therefore, was unsuitable for soil assays for $V$. dahliae. However, NP-10 with P-3889 and concentrations of $\mathrm{NaOH}$ from 0.015 to $0.025 \mathrm{~N}$ improved the recovery of MS from soil and mycelial growth on the medium. Furthermore, the size of the MS was identical to that observed on NP-10 with P-1879, which is no longer available. Concentration of $\mathrm{NaOH}$ either below or above this range in the NP10 containing P-3889 decreased the growth of $V$. dahliae and the recovery of MS from soils. It is likely that the $\mathrm{pH}$ of the media regulated by $\mathrm{NaOH}$ may have influenced the growth and recovery of $V$. dahliae. Increased $\mathrm{pH}$ in these media with high concentrations of $\mathrm{NaOH}$ also may have prevented the growth and recovery of $V$. dahliae as demonstrated previously on $V$. marguandi (16) and $V$. dahliae (19). A pH $<6.0$ yielded optimum mycelial growth of

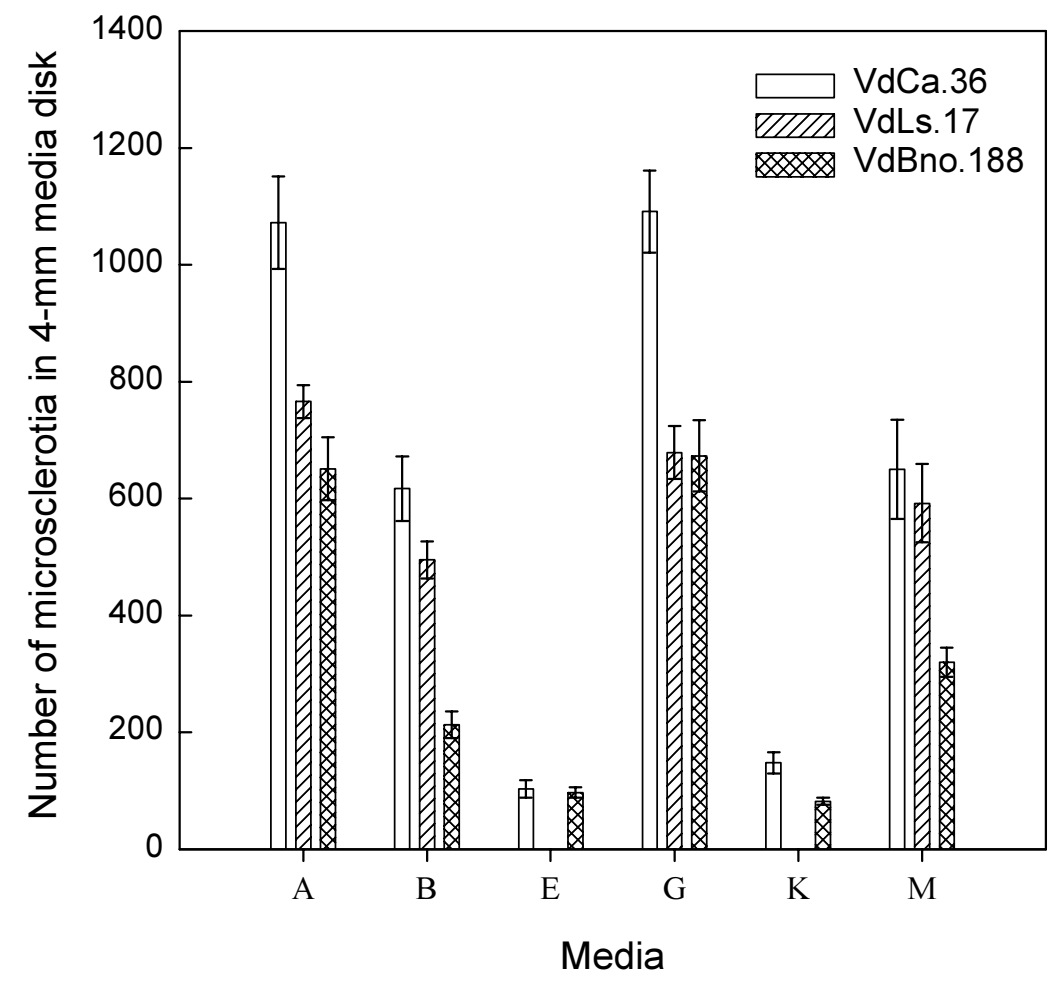

Fig. 3. Number of microsclerotia (in 4-mm agar plug) of Verticillium dahliae isolates from chili pepper (VdCa.36), lettuce (VdLs.17), and oilseed rape (VdBno.188) on different media: A, NP-10 with P-1879; B, NP-10 with P-3850; E, NP-10 with P-3889 and 0.015 N NaOH; G, NP-10 with P-3889 and $0.025 \mathrm{~N} \mathrm{NaOH}$; K, NP-10 with P-3889 and $0.055 \mathrm{~N} \mathrm{NaOH}$; and M, Na-pectate. Vertical bars represent the standard errors of the mean. 


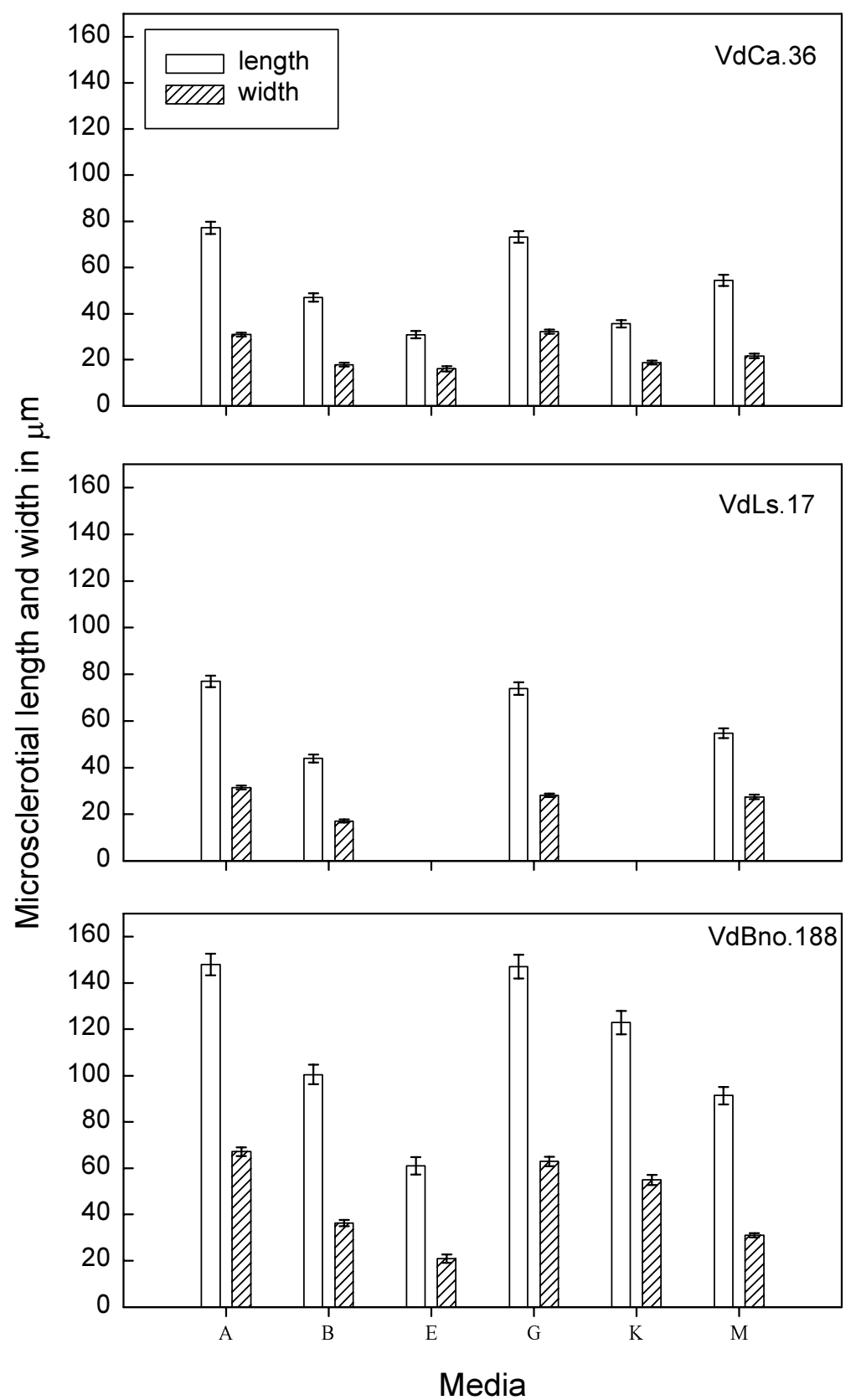

Fig. 4. Length and width of microsclerotia of Verticillium dahliae isolates from chili pepper (VdCa.36), lettuce (VdLs.17), and oilseed rape (VdBno.188) on different media: A, NP-10 with 1879; B, NP-10 with P-3850; E, NP-10 with P-3889 and 0.015 N NaOH; G, NP-10 with P-3889 and $0.025 \mathrm{~N} \mathrm{NaOH}$; K, NP-10 with P-3889 and $0.055 \mathrm{~N} \mathrm{NaOH}$; and M, Na-pectate Vertical bars represent the standard errors of the mean.

V. marguandi (16). In our study, suitable $\mathrm{pH}$ for mycelial growth of $V$. dahliae ranged from 4.81 to 5.03. Even though the $\mathrm{pH}$ of NP-10 with P-3889 alone was 2.63, growth of $V$. dahliae was inhibited, suggesting that addition of $\mathrm{NaOH}$ in the medium is essential to increase the $\mathrm{pH}$ of the media to an optimum level for the growth of $V$. dahliae. It also is possible that the $\mathrm{Na}^{+}$ion itself may have been toxic to the pathogen at higher concentrations of $\mathrm{NaOH}$. The high concentration of $\mathrm{NaOH}$ $(0.03$ to $0.055 \mathrm{~N})$ in the NP-10 media also changes the color of the media from light to dark brown, which may reduce the contrast between MS colonies and the media, gesting the requirement for NP-10 with either P-1879 or P-3889 and $0.025 \mathrm{~N}$ $\mathrm{NaOH}$ for soil assays of $V$. dahliae MS.

Mycelial growth was slower on the cellophane medium than on NP-10 with P1879 or P-3889 and $0.025 \mathrm{~N} \mathrm{NaOH}$. Microsclerotial recovery from the soils by the cellophane medium was significantly higher than on Na-pectate medium in all soils but lower than on the NP-10 with P1879 or P-3889 and $0.025 \mathrm{~N}$ in six soils. However, in one soil (soil 1, Table 1) with the lowest number of MS in our study, recovery of MS on cellophane medium was not significantly different from that on NP-10 containing P-1879, suggesting that cellophane medium could be useful in soils containing low densities of $V$. dahliae. One advantage of the cellophane medium, however, is its ability to distinguish $V$. dahliae colonies from $V$. tricorpus (1). However, cellulolytic microorganisms outgrew $V$. dahliae colonies and led to an underestimation of the number of MS. In contrast, growth of other fungal colonies on the NP10 and Na-pectate media was slow and restricted; therefore, MS colonies were easily detectable under the microscope. Different types of cellophane have the potential to affect the growth and recovery of $V$. dahliae from soils. We tested three kinds of cellophane, such as cellophane (1), gel dryer cellophane membrane (BioRad Laboratories, Hercules, CA), and an unknown type of cellophane. Neither the growth of different isolates of $V$. dahliae nor the recovery of MS from the soil was affected by the types of cellophane tested (data not shown), indicating that the type of cellophane may not be a major factor determining the growth and recovery of $V$. dahliae.

The amount of soil plated on the media can influence the accurate estimation of $V$. dahliae MS. Distributing $33 \mathrm{mg}$ of soil in each plate failed to provide an accurate count of $V$. dahliae MS in soil. A small error in spreading this small amount of soil on plates may cause a large variation in the estimation of MS in both low- and highdensity $V$. dahliae MS in soil. In contrast, a large amount of soil $(200 \mathrm{mg}$ ) plated on the medium increased the number of the MS colonies to levels where an accurate counting of the number of colonies was difficult. Plating $100 \mathrm{mg}$ of soil per plate was optimal for an accurate estimation of MS colonies, and this was consistent with the conclusions of the study by Butterfield and DeVay (3).

Microsclerotia of Verticillium spp. germinate repeatedly in soil (4). Nearly $60 \%$ of the MS recovered from the soil assayed on NP-10 with P-1879 or P-3889 and $0.025 \mathrm{~N} \mathrm{NaOH}$, further confirming the ability of NP-10 with P-3889 and $0.025 \mathrm{~N}$ $\mathrm{NaOH}$ to replace NP-10 with P-1879. Huisman and Ashworth (7) reported no significant reduction in germination between Na-pectate and cellophane media. In this 


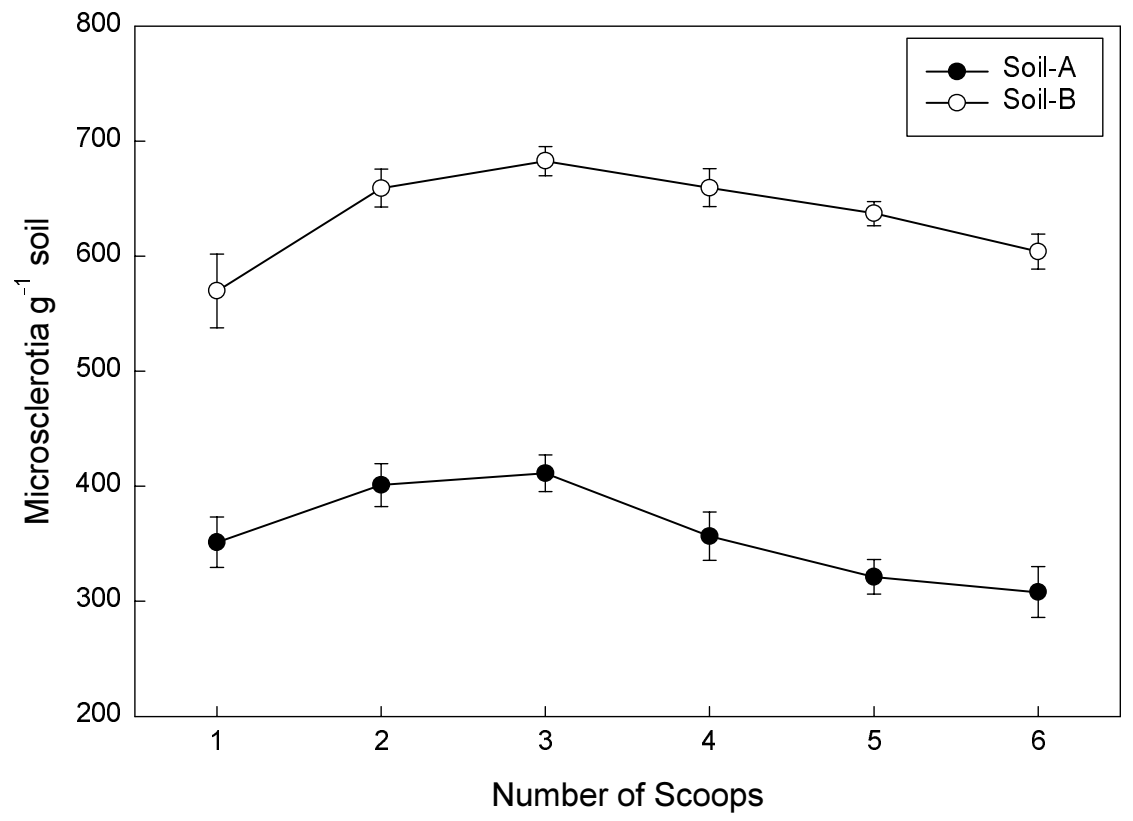

Fig. 5. Relationship between the amount of soil plated using the Anderson sampler technique and the number of Verticillium dahliae microsclerotia recovered in two soils with different levels of infestation by the pathogen. Vertical bars represent the standard errors of the mean.

study, however, germination of MS was significantly lower in the cellophane medium relative to that in Na-pectate medium.

Our results demonstrate that the PGA from orange (P-3889) amended with 0.025 $\mathrm{N} \mathrm{NaOH}$ in the NP-10 is a suitable alternative to P-1879 (the erstwhile constituent of NP-10 semiselective medium). The $\mathrm{pH}$, recovery of MS from soils, size of MS and their germination, and the growth of $V$. dahliae on these media were similar. Compared with the NP-10 with P-1879 or P3889 amended with $0.025 \mathrm{~N} \mathrm{NaOH}, V$. dahliae grew more slowly on the NP-10 with P-3850 and on the cellophane medium for all isolates. Recovery of MS of $V$. dahliae was higher on the NP-10 with $\mathrm{P}-1879$ or P-3889 amended with $0.025 \mathrm{~N} \mathrm{NaOH}$ than on NP-10 with P-3850 or Na-pectate medium for all tested soils.

\section{ACKNOWLEDGMENTS}

We thank S. T. Koike for providing original polygalacturonic acid (P-1879), O. C. Huisman for providing Na-pectate, and M. E. Abarca and M. Orozco for their technical assistance.

\section{LITERATURE CITED}

1. Ashworth, L. J., Jr., Waters, J. E., George, A. G., and McCutcheon, O. D. 1972. Assessment of microsclerotia of Verticillium albo-atrum in field soils. Phytopathology 62:715-719.

2. Bhat, R. G., and Subbarao, K. V. 1999. Host range specificity in Verticillium dahliae. Phytopathology 89:1218-1225.

3. Butterfield, E. J., and DeVay, J. E. 1977. Reassessment of soil assays for Verticillium dahliae. Phytopathology 67:1073-1078.

4. Farley, J. D., Wilhelm, S., and Snyder, W. C. 1974. Repeated germination and sporulation of microsclerotia of Verticillium albo-atrum in soil. Phytopathology 61:260-264.

5. Grogan, R. G., Ioannou, N., Schneider, R. W., Sall, M. A., and Kimble, K. A. 1979. Verticillium wilt on resistant tomato cultivars in California: Virulence of isolates from plants and soil and relationship of inoculum density to disease incidence. Phytopathology 69:1176-1180.

6. Harris, D. C., and Yang, J. R. 1996. The relationships between the amount of Verticillium dahliae in soil and the incidence of strawberry wilt as a basis for disease risk prediction. Plant Pathol. 45:106-114.

7. Huisman, O. C., and Ashworth, L. J., Jr. 1974. Quantitative assessment of Verticillium alboatrum in field soils: Procedural and substrate improvements. Phytopathology 64:1043-1044.

8. Jordan, V. W. 1974. Verticillium wilt of strawberry: cultivar reaction and effect on runner health and production. Plant Pathol. 23:8-13.

9. Kabir, Z., Bhat, R. G., and Subbarao, K. V. 2001. Optimizing polygalacturonic acid in NP-10 medium to improve Verticillium dahliae recovery from soil (Abstr.) Phytopathology $91: 45$.

10. Nicot, P. C., and Rouse, D. I. 1987. Precision and bias of three quantitative soil assays for Verticillium dahliae. Phytopathology 77:875-881.

11. Nnudo, E. C., and Harrison, M. D. 1979. The relationship between Verticillium albo-atrum inoculum density and potato yield. Am. Potato J. 56:11-25.

12. Paplomatas, E. J., Bassett, D. M., Broome, J. C., and DeVay, J. E. 1992. Incidence of Verticillium wilt and yield losses of cotton cultivars (Gossypium hirsutum) based on soil inoculum density of Verticillium dahliae. Phytopathology 82:1417-1420.

13. Pegg, G. F. 1984. The impact of Verticillium diseases in agriculture. Phytopathol. Mediterr. 23:176-192.

14. Schnathorst, W. C. 1981. Life cycle and epidemiology of Verticillium. Pages 81-111 in: Fungal Wilt Diseases of Plants. M. E. Mace, A. A. Bell, and C. H. Beckman, eds. Academic Press, Inc., New York.

15. Sorensen, L. H., Scheider, A. T., and Davis, J. R. 1991. Influence of sodium polygalacturonate sources and improved recovery of Verticillium spp. from soil (Abstr.) Phytopathology $81: 1347$.

16. Staba, M., and Dtugonski, J. 2000. Selective recovery of $\mathrm{Zn} 2+$ from waste slag from a metal-processing plant by the microscopic fungus Verticillium marquandii. Biotechnol. Lett. 22:1699-1704.

17. Subbarao, K. V., Chassot, A., Gordon, T. R., Hubbard, J. C., Bonello, P., Mulin, R., Okamoto, D., Davis, R. M., and Koike, S. T. 1995. Host range of Verticillium dahliae from cauliflower and genetic relationships and cross pathogenicities of isolates from different crops. Phytopathology 85:1105-1112.

18. Termorshuizen, A. J., Davis, J. R., Gort, G., Harris, D. C., Huisman, O. C., Lazarovits, G., Locke, T., Melero Vara, J. M., Mol, L., Paplomatas, E. J., Plat, H. W., Powelson, M., Rouse, D. I., Rowe, R. C., and Tsror, L. 1998. Interlaboratory comparison of methods to quantify microsclerotia of Verticillium dahliae in soil. Appl. Environ. Microbiol. 64:3846-3853.

19. Wheeler, T. A., Madden, L. V., Rowe, R. C., and Riedel, R. M. 1992. Modeling of yield loss in potato early caused by Pratylenchus penetrans and Verticillium dahliae. J. Nematol. 24:99-102.

20. Wilhelm, S. 1955. Longevity of Verticillium wilt fungus in the laboratory and the field. Phytopathology 45:180-181.

21. Xiao, C. L., and Subbarao, K. V. 1998. Relationships between Verticillium dahliae inoculum density and wilt incidence, severity and growth of cauliflower. Phytopathology 88:1108-1115. 